\title{
Artificial Intelligence Approach in Multiclass Diabetic Retinopathy Detection Using Convolutional Neural Network and Attention Mechanism
}

\author{
Amnia Salma ${ }^{1}$, Alhadi Bustamam ${ }^{1 *}$, Anggun Rama Yudantha ${ }^{2}$, Andi Arus \\ Victor $^{2}$, and Wibowo Mangunwardoyo ${ }^{3}$ \\ ${ }^{1}$ Department of Mathematics, Faculty of Mathematics and Natural Science, \\ Indonesia University, Depok, West Java, 16424, Indonesia \\ e-mail: amniasalma@ssci.ui.ac.id \\ *Corresponding author, e-mail: alhadi@ssci.ui.ac.id \\ ${ }^{2}$ Department of Opthalmology, Faculty of Medicine, Indonesia University, Cipto \\ Mangunkusumo National Hospital, Jakarta, Indonesia \\ ${ }^{3}$ Department of Biology, Faculty of Mathematics and Natural Science, Indonesia \\ University, Depok, West Java, 16424, Indonesia
}

\begin{abstract}
The number of people around the world who have diabetes is about 422 million. Diabetes seriously affects the blood vessels in the retina, a disease called diabetic retinopathy $(D R)$. The ophthalmologist examines signs through fundus images, such microaneurysm, exudates and neovascularisation and determines the suitable treatment for patient based on the condition. Currently, doctors require a long time and professional skills to detect DR. This study aimed to implement artificial intelligence (AI) to resolve the lack of current methods.

This study implemented AI for detecting and classifying DR. AI uses deep learning, such the attention mechanism algorithm and AlexNet architecture. The attention mechanism algorithm focuses on detecting the pathological area in the fundus images, and AlexNet is used to classify DR into five levels based on the pathological area. This study also compared AlexNet architecture with and without attention mechanism.
\end{abstract}

We obtained 344 fundus images from the Kaggle dataset, which contains normal, mild, moderate, severe and proliferative DR. The 


\begin{abstract}
highest accuracy in this study is up to $91 \%$ and used the attention mechanism algorithm and AlexNet architecture. The experiment shows that our proposed method can provide results that can detect the pathological areas and effectively classify DR.
\end{abstract}

Keywords: Artificial intelligence, Diabetic Retinopathy, Attention Mechanism, AlexNet

\title{
1 Introduction
}

According to data from the World Health Organization 2020, diabetes cases increase yearly. In 1980, the total number of patients with diabetes was 180 million, and in 2020, it has increased to 422 million [1]. People with diabetes are prone to diabetic retinopathy (DR) an eye disease where high blood sugar levels cause damage to blood vessels in the retina. These blood vessels can swell and leak or they can close, which impedes blood from passing through, and sometimes, abnormal new blood vessels grow on the retina [2]. All these changes can cause blindness. One of the early signs of DR is microaneurysms, which are little red spots in the retina [3]. The other signs are haemorrhage or red lesions and hard exudates, which occur due to the breaking of vessels and breakdown of the blood-retina barrier, respectively, allowing the leakage of serum protein and lipids from vessels [4,5]. In severe DR, neovascularisation occurs, which is a hallmark of proliferative diabetic retinopathy (PDR), where new vessels are commonly seen along the retinal arcades and can occur at the optic disc [6]. Early detection is needed for suitable treatments so that serious damage and blindness can be prevented. Currently, doctors must look at fundus images one by one and based on the condition in the fundus image, can diagnose patients as normal, mild, moderate, severe and PDR. However, this method requires a long time and professional skills to examine DR. Many researchers developed several applications in medicine to resolve the lack of current methods on DR detection [7], and one of the well-known applications used is artificial intelligence (AI).

The application of AI for DR detection help the opthalmologist to do the task easier. Currently, many developments of AI, there are Machine Learning, Deep Learning, and Convolutional Neural Network (CNN). CNN is used to detect DR disease through fundus images into normal, mild, moderate and severe. Beside focusing on classification, we also focusing on the pathological area, so we implementat the Attention Mechanism to help CNN for better performance.

In this study, we proposed two algorithms such $\mathrm{CNN}$ to focus on classification and Attention Mechanism to focus on pathological area, these methods used to detection and classification of DR using fundus images. We also compare the performance of $\mathrm{CNN}$ with Attention Mechanism and performance of $\mathrm{CNN}$ without Attention Mechanism. 


\section{Related Work}

Detection of disease has been the focus of AI since approximately in the 1970s until the present. Many diseases, such as DR, have been detected because AI uses methods similar to that of humans in learning data. AI is used for DR detection because it is rapid in detecting DR, making the task of ophthalmologists easier [8].

One of the methods used by AI is Deep Learning (DL), which could improve the performance of object detection and visual object recognition [9]. Deep Learing is a subset of machine learning that uses multiple layers containing non-linear processing units. DL application uses object detection of features of images, such as lesion features in the fundus images, for detecting DR [10]. The convolutional neural network $(\mathrm{CNN})$ is one of the DL models that have convolutional, pooling and fully connected layers. The convolutional layer extracts image features, the result of which goes to the pooling layer where the number of feature extraction is calculated [12]. The fully connected layer is classified as a DL algorithm [13,14].

Many researchers used CNN for DR detection, and have [15] detected normal class DR. DR class used AlexNet architecture and add normalisation in the preprocessing images by 200 images fundus achieved 90\% accuracy. In other research on DR detection, [16] AlexNet architecture was used and detection was divided into three classes, normal, non-proliferative DR and PDR. The result of this research achieved an accuracy of up to $88 \%$, with a total data of 1,000 fundus images. The development of research for DR detection divided the classes into mild, moderate, severe and PDR and this research achieved accuracy of up to $75 \%$ [17].

One of the algorithms helped CNN for better accuracy to classify images is attention mechanism. Attention mechanism is an algorithm, which contains three steps for implementation, such as global, local and fusion branches [18]. Attention mechanism focuses on the pathological area after fundus images are divided into normal and DR and into four class, mild, moderate, severe and PDR.

In this study, we proposed attention mechanism and $\mathrm{CNN}$ as methods for the detection and classification of DR using fundus images. We used AlexNet as an architecture of CNN because AlexNet is the fastest; we used AM because it can focus on the pathological area, and AlexNet was used for classify DR into normal, mild NPDR, moderate NPDR, severe NPDR and PDR. This research also used AlexNet architecture without attention mechanism for detecting and classifying DR using fundus images. We compared the results between AlexNet with and without attention mechanism. 


\section{Problem Formulations or Methodology}

In recent years, the development of applied AI for medicine has been challenging for researchers. In the big data era, many methods, such as CNN (AlexNet architecture), are used to classify images for disease detection. In this research, we used the AlexNet architecture and attention mechanism algorithm to classify fundus images into DR classes based on the symptom shown. The steps of our experiment shown on Fig.1.

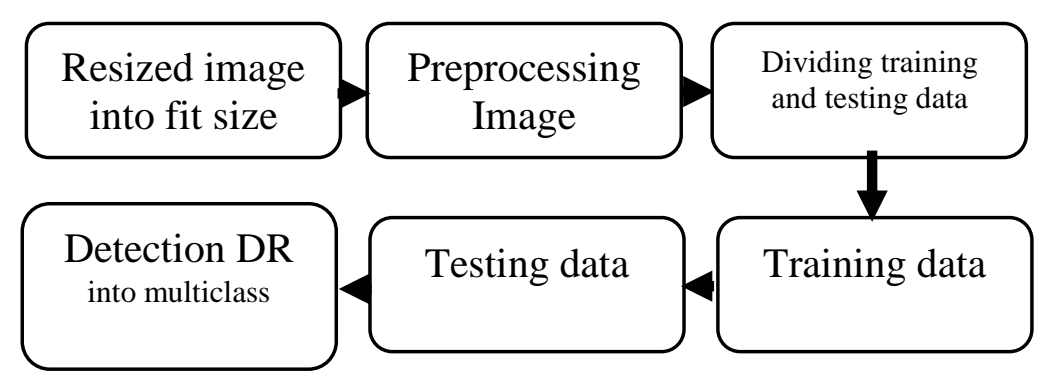

Figure 1. Flowchart DR Classification

First step we resize the image into $227 \times 227$ as AlexNet fit input size. The we preprocessing the data through augmentation data. After preprocessing, the data divided into training dan testing. After data division we perform the model through training and testing proccess. After the performance of model, we get the result of DR classification.

In the performance of model, we implement the Attention Mechanism to help CNN getting better result. We divided the step into three parts. First part called global branch, second part called local branch and the last part called fussion branch.

\section{The Proposed Method}

Our model, AlexNet and AlexNet architecture with attention mechanism training 344 fundus images from the Kaggle dataset to classify normal, mild, moderate, severe and PDR. Before training data, we did image pre-processing when the images resize into fit AlexNet input image. By this process, we know the weight of each input. Afterwards, we trained the data using AlexNet architecture and AlexNet architecture with attention mechanism to know the classification result. Zero indicates normal fundus images, 1 indicates mild fundus images, 2 indicates moderate fundus images, 3 indicates severe fundus images and 4 indicates PDR fundus images. The proposed method in this research used several steps. The first step is to pre-process data, including the augmentation and resizing of data into a fit size. We used CNN models, such as AlexNet and attention mechanism 
algorithm, for training data. AlexNet has a faster performance for training data, and attention mechanism algorithm helps the AlexNet to classify detailled data.

We used fundus images containing normal, mild, moderate, severe and PDR for training data. We obtained database from the Kaggle dataset, which consists of 344 images that we used for training the model. The fundus images were divided into 70 normal fundus images, 70 mild fundus images, 65 moderate fundus images, 69 severe fundus images and 70 PDR fundus images. We built the model to classify DR into five levels, normal, mild, moderate, severe and PDR. Normal or No DR means that the eye is healthy and there are no DR symptoms; mild means there are microaneurysms; moderate means that the number of microaneurysms and haemorrhages is less than 20 in every quadrant as well as the presence of hard exudates; severe means that the number of microaneurysms and haemorrhages is more than 20 in more than two quadrants as well as exudates and red lesions; PDR means that neuvascularisation, abnormal formation of blood vessels, have developed, as evident in the fundus images.

We set the image into fit size for by resized each fundus images. Every fundus image was pre-processed and the data was divided to $80 \%$ for training data and $20 \%$ for testing data. We used the AlexNet architecture and AlexNet architecture with attention mechanism algorithm to train and test data. The output of this research is the classification of DR into normal, mild, moderate, severe and PDR.

\subsection{Dataset}

Obtaining dataset is one of the challenging parts of this study. There are many online databases, and one of them is Kaggle, which has a lot of fundus image datasets with a high resolution (https://www.kaggle.com). The images in the Kaggle dataset are indexed by the pathologist. We used the fundus images (normal and DR) from the Kaggle dataset.

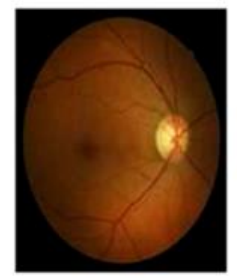

Sormul

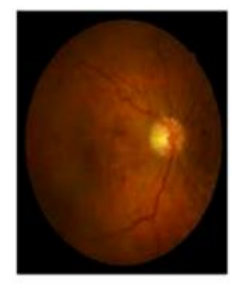

Severe

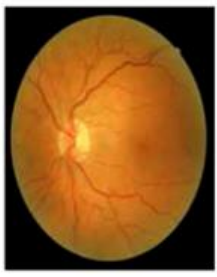

Mild

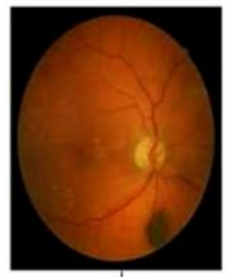

Moderate

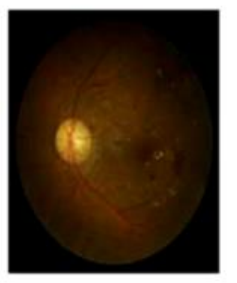

Proliferative

Figure 2. DR in five severe levels [19] 
Sample of a fundus image (normal, mild NPDR, moderate NPDR, severe NPDR and PDR criteria shown in Fig.2) number of datasets is shown in Table 1. We have 344 fundus images from the dataset and the data was divided into $80 \%$ for training data and $20 \%$ for data validation.

Table 1. datasets division

\begin{tabular}{l|l|l|l}
\hline & Training Data & Testing Data & Data \\
\hline Normal Fundus Images & 56 & 14 & 70 \\
Mild Fundus Images & 56 & 14 & 70 \\
Moderate Fundus Images & 52 & 13 & 65 \\
Severe Fundus Images & 55 & 14 & 69 \\
PDR fundus Images & 56 & 14 & 70 \\
Total & 275 & 69 & 344 \\
\hline
\end{tabular}

We provided the balance data with the categories, normal, mild and PDR (70 fundus images). Moderate fundus images are 65, and severe fundus images are 69. We estimated that fundus images data have almost the same total number with little difference.

\subsection{Image Pre-processing}

We used 200 images fundus dataset, such 39 normal fundus and 161 DR fundus. The size of each image is 3152 x 3000 or approximately $9 \mathrm{M}$ in size with the type is .jpg format. The images are resized to fit ALexNet architecture properly. The valid image size for AlexNet is $227 \times 227$ pixels. The type color of image is RGB images because RGB are complex to process and requires a lot of time, more number of features can be extracted from such images.

\subsection{Image Augmentation}

In this research, we also used image augmentation to improve the localisation ability of the network. The original images were only used for training once. We augmented the images with random horizontal and vertical flips.

\subsection{Convolutional Neural Network}

Convolutional Neural Network $(\mathrm{CNN})$ is a part of DL, which has many architectures to classify image, text, sound, video, etc. There are several variations on CNN layers, such as the convolutional, pooling and fully connected layers $[20,21]$. CNN is a part of the detection images which computer has never seen before [22,23]. In the convolutional layer, if a particular dataset of images is given to train, CNN first multiplies the pixel values with the selected stride as the hyperparameter. This layer extracts the features from the images using multiple 
features. The earlier standard in traditional neural networks for the non-linear part was a function of Tanh or Sigmoid. The CNN architecture is shown in Fig.3.

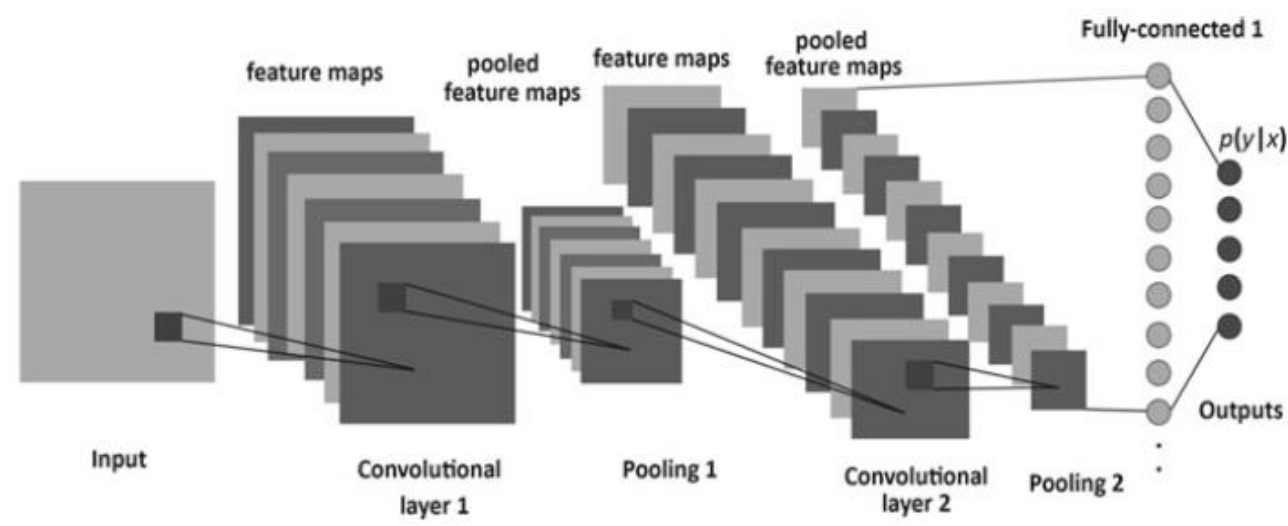

Figure 3. CNN architecture [21]

CNN has three layers such Convolutional Layer, Pooling and Fully Connected Layer $[24,25]$. The convolutional layer is the first layer on CNN the features are extracted by the system. ReLu is a unit layer that deactivates the unnecessary pixels and only keeps the important pixels. The pooling layer reduces the input size. The fully connected layer has multiple neurons and connects neurons to each other. It takes the input as numbers from previous layers and feeds the data to the neural network $[26,27]$.

\subsection{AlexNet}

In this research we used AlexNet for classify because its work fast and accurate for image classification. We used AlexNet with five convolutional layers and three fully connected layers for classification. The layers are the convolution layer, pooling layer, activation function and Fully Connnected layer [28,29]. Then in the AlexNet the next features are ReLu (Rectified Linear Unit), it trains faster than the latter. ReLu value is computed by,

$$
f(x)=\max (0, x)(1)
$$

The training process in the pooling layer further help convert negative pixels to positive ones and shrink image size to lower levels. In AlexNet, to reduce the over-fitting problem, we used the DropOut layer. In this research, AlexNet was used for image classification because it is fast and accurate. The AlexNet architecture is shown on Fig.4. 


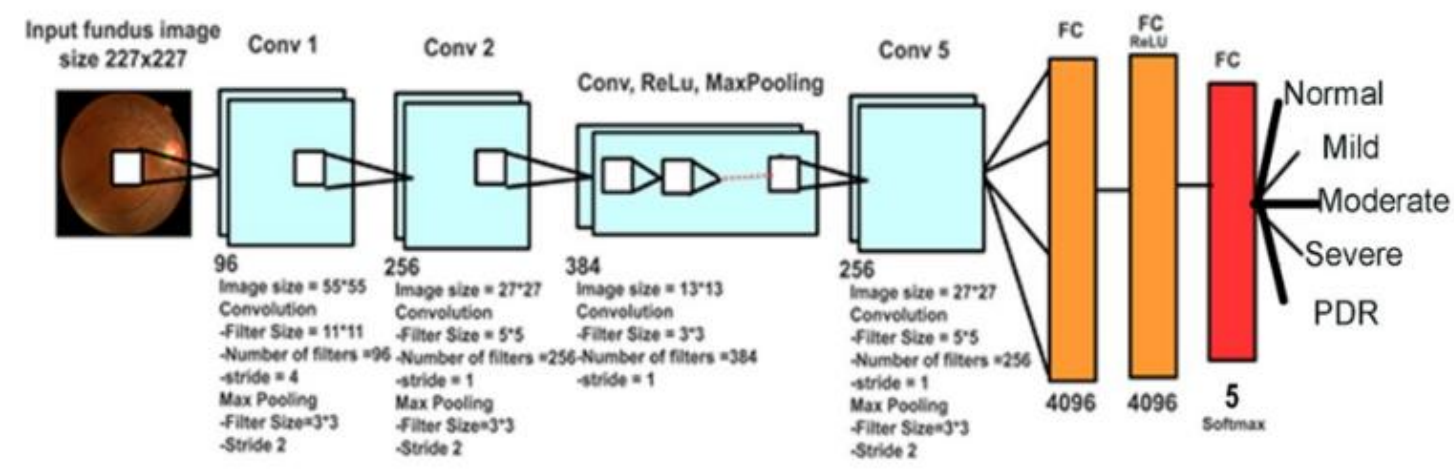

Figure 4. Alexnet Architecture

We used AlexNet architecture for this model. First the neural network trained 275 images from the Kaggle dataset, then another 69 images was used to test. For image processing, the image was resized to fit AlexNet $227 \times 227$ pixels and divided into multiple 227 x 227 pixels. Every piece was given specific weight where the area in the centre of the eye has highest weight. The value of a higher weight represents more intensity of the disease; thus, the fundus with a higher weight was indicated as DR. We trained the model using Python programming, Pytorch and Tensorflow libraries. Every piece is input to the model, which has 96 hidden layers in the first convolutional layer and 256 hidden layers in the second convolutional layer, 384 hidden layers in the third convolutional layer and 256 hidden layers in the fourth and fifth convolutional layers. Every convolutional layer is followed by max pooling and activation function. In the fully connected layer, we have 4096, followed by the second fully connected layer, ReLu layer, the fully connected layer and softmax. After this process, we can classify the image into five class, normal, mild, moderate, severe and PDR.

\subsection{AlexNet and Attention Mechanism Algorithm}

Fig. 5 shows the implementation of the attention mechanism to AlexNet in this research. After pre-processing data, fundus images in the first output through convolution and max pooling. The function of this process is to extract the data and determine normal and DR fundus images. After that, the part of concenate is used to connect the result of the first output and input images into second process such AlexNet architecture without fully connected layer and at the end process we add deconvolution to fit the size for the next process. In the last process, we used concenate again to connect the second process to the last process. The last process used AlexNet architecture and we obtained the classification of multiclass. 


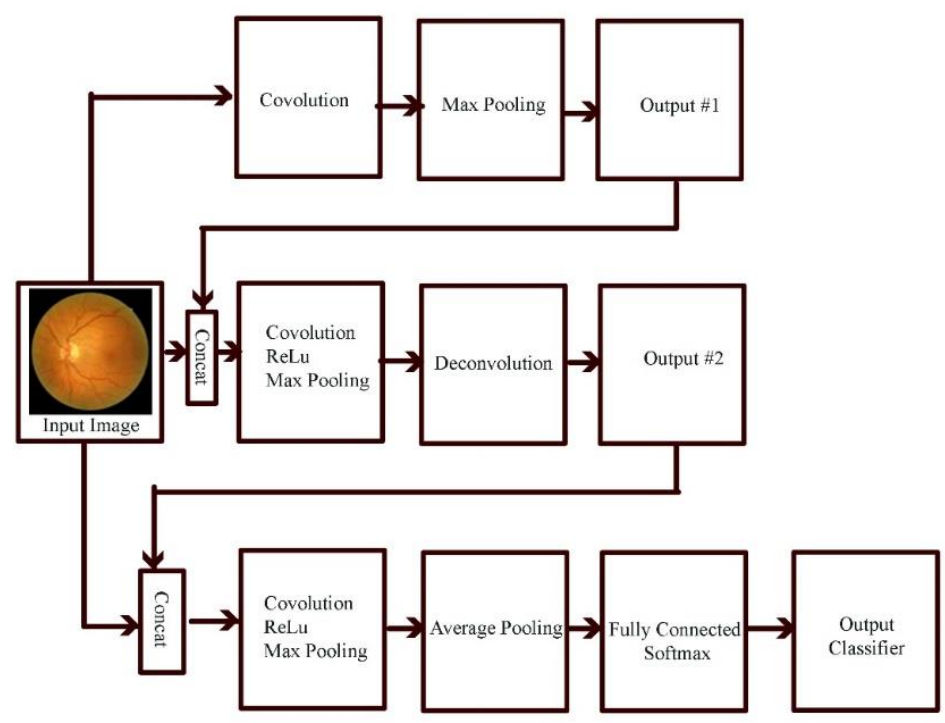

Figure 5. Alexnet Architecture and Attention Mechanism

Attention Mechanism consists of three points, global branch, local branch and fussion branch. First, we have to set label each image. Given label in the every images into vector for $L=\left[l_{1}, l_{2}, \ldots l_{c}\right]$ where $l_{c} \in\{0,1\}$ and $l_{c}$ represent pathological area. Global branch denoted by,

$$
p_{g}(C \mid I)=\frac{1}{1+\exp (-p(C \mid I))}
$$

Where I represents global image $p_{g}(C \mid I)$ is a probability of I for the class C. Then for parameter optimation used to minimum of Binary cross entropy (BCE) loss, denoted by,

$$
L\left(W_{(g)}\right)=-\frac{1}{C} \sum_{C=1}^{C} l_{c} \log \left(P_{(g)}(c \mid I)\right)+\left(1-l_{(c)}\right) \log \left(1-P_{(g)}(c \mid I)\right)
$$

Local branch denoted by,

$$
L\left(W_{(I)}\right)=-\frac{1}{C} \sum_{C=1}^{c} l_{c} \log \left(P_{(g)}(c \mid I)\right)+\left(1-l_{c}\right) \log \left(1-P_{(g)}\left(c \mid I_{c}\right)\right)
$$

And then Fusion Branch denoted by,

$$
L\left(W_{f}\right)=-\frac{1}{C} \sum_{C=1}^{c} l_{c} \log \left(P_{f}\left(c \mid\left[I, I_{c}\right]\right)\right)+\left(1-l_{c}\right) \log \left(1-P_{f}\left(c \mid\left[I, I_{c}\right]\right)\right)
$$

The role of attention mechanism on the AlexNet architecture is shown in three division learning processes. The first learning process has the result of data which used as an input data for second process and used to know the DR area and normal area. In the second process, we classified the DR area into several levels. All DR levels become output in the last process using softmax. 


\section{Results, Analysis and Discussions}

In this research, we used Google Collaboratory and Python programming language along with Pytorch library to simulate the model with 344 fundus images obtained from the Kaggle dataset shown in Fig.2. The result of our model is shown in Table 2 .

Table 2. The performance of AlexNet and Attention Mechanism.

\begin{tabular}{l|l|c}
\hline & AlexNet & $\begin{array}{c}\text { AlexNet and } \\
\text { Attention Mechanism }\end{array}$ \\
\hline Input image size & $224 \times 224 \times 3$ & $224 \times 224 \times 3$ \\
Average Accuracy & $76 \%$ & $91 \%$ \\
Average of Running time & 15 seconds & 13 seconds \\
\hline
\end{tabular}

The input size of fundus images is the same between AlexNet architecture and AlexNet with attention mechanism. AlexNet with attention mechanism shows better accuracy than AlexNet architecture. The difference accuracy of both models is $15 \%$, with $76 \%$ on AlexNet architecture and $91 \%$ for AlexNet with attention mechanism. AlexNet is faster than AlexNet with attention mechanism in terms of runtime. For epoch training both models had 40 epochs. Every models iterates four times.

\subsection{AlexNet}

A total of 20 min was needed for training with 20 epochs. After 40 epochs of training the images, the accuracy of the result significantly increased to $76 \%$. The model trained on the full dataset of 275 images for 40 epochs, and the time for training was fast. We used 0,001 learning rate and adam as optimiser. Our model suffers from over-fitting. To solve the over-fitting, we set the input parameter on convolutional architecture of AlexNet from to default input shown on Fig.4.

\subsection{AlexNet and Attention Mechanism Algorithm}

The normal class indicates no disease, and the DR class indicates an infected or diseased eye. The model was pre-trained on 344 datasets until it reached a significant level. A total of $20 \mathrm{~min}$ was needed for training with 40 epochs. After 80 epochs of training the images, the accuracy of the result getting significantly increases from $42 \%$ to $91 \%$. The model trained on the full dataset of 275 images for 80 epochs. To prevent the over-fitting, we set the input parameter architecture of AlexNet from customised to default.

AlexNet architecture was obtained from Pytorch library. The AlexNet was trained using adam optimiser. A low learning rate of 0,001 was used for 80 epochs to stabilise the weight of each image input.

\section{Conclusion}

This study represents a new model to classify DR. Using AlexNet architecture, the result gives an accuracy of $76 \%$. In the medical field, future research is necessary 
to get better accuracy. In this study, we started the model to implement DL using fundus images.

AlexNet with attention mechanism algorithm showed very good results of learning the feature required to classify the fundus images. The advantages of using AlexNet with attention mechanism algorithm for detecting DR using fundus images trained the model with an efficient memory and low computations to perform. By using Google Collaboratory, the performance can increase quickly because of GPU usage. Our model makes a fast diagnosis and correct detection and classification for the patient, achieving an accuracy of $91 \%$.

In conclusion, we have shown that $\mathrm{CNN}$ has the potential training to identify DR features using fundus images. This study represents a new model for DR detection and classification (normal, mild, moderate, severe and PDR). Using AlexNet architecture from $\mathrm{CNN}$ and attention mechanism algorithm, results are $91 \%$ accurate. In this study, we started the model to implement DL using fundus images. Besides, this system was created to help ophthalmologists detect DR accurately and timely.

In the future, we have plans to classify DR into five classes such every level on DR can detect correctly and CNN trained focus not only detect normal and DR fundus images but also the classify of DR level from mild, moderate, severe and PDR. Furthermore, we plan to obtain dataset from a real Indonesia screening setting, such as a hospital in Indonesia that specialises in eye treatment. The ongoing development of combining CNN with other support algorithms allow deeper networks to learn the data getting better.

\section{ACKNOWLEDGEMENTS}

We express our gratitude to Universitas Indonesia, Faculty of Mathematics and Natural Science, who funded this research through PDUPT grant Number: NKB-183/UN2.RST/HKP.05.00/2021

\section{References}

[1] WHO. Diabetes. 30 Oktober 2018 https://www.who.int/news-room/factsheets/detail/diabetes. diakses pada 21 Desember 2019.

[2] Cheung, Ning., Paul Mitchell, Tien Yin Wong. Daibetic Retinopathy. The Lancet, Vol. 376. 2010

[3] Indumathi, G., \& V. Sathananthavath. Microaneurysms Detection for Early Diagnosis of Diabetic Retinopathy Using Shape and Steerable Gaussian Features. Telemedicine Technologies, 57-69. 2019.

[4] Joshi, Shilpa \& P.T. Karule. A review on exudates detection methods for diabetic retinopathy. Jurnal Biomedicine \& Pharmacotherapy. ELSEVIER, 1454-1460. 2018. 
[5] Khojasteh, Parham., Behzad Aliahmad \& Dinesh K. Kumar. Fundus images analysis using deep features for detection of exudates, hemorrhages and microaneurysms. BMC Ophthalmology, 275-288. 2018.

[6] Mahiba, C. \& A. Jayachandran. Severity analysis of diabetic retinopathy in retinal images using hybrid structure descriptor and modified CNNs. ELSEVIER, 762-767. 2019

[7] Schmidt-Erfurth, Ursula., Amir Sadeghipour., Bianca S. Garendas., Sebastian M. Waldstein, Hrvoje Bagunovic. Artificial Intelligence in Retina. Progress in Retinal and Eye Research. Vol. 67, 1-29. 2018

[8] Davenport, T., \& Kalakota, R. The potential for artificial intelligence in healthcare. Future healthcare journal, 6(2), 94-98. https://doi.org/10.7861/futurehosp.6-2-94. 2019.

[9] Abdillah, Bariqi., Alhadi Bustamam, Devvi Sarwinda. Classification of Diabetic Retinopathy Through Texture Features Analysis. ICACSIS, 333339. 2017.

[10] Lecun, Y. Bengio and G. Hinton. "Deep Learning”, nature, vol. 521, no. 7553, 436-444. 2015

[11] Li, Zhuo ling., Minghui Dong, Shiping Wend, Xiang Hub. Pan Zhou \& Zhigang Zeng. CLU-CNNs: Object detection for medical images. ELSEVIER, 53-59. 2019

[12] Biradjar, U., Gadhave, S., Chikodikar, S., Dadich, S., Chiwhane, S., Detection and Classification of Diabetic Retinopathy Using AlexNet Architecture of Convolutional Neural Networks. Proceeding of International Confernce on Computational Science and Applications. 2019.

[13] Kharabe S, Nalini C. Using adaptive thresholding extraction-robust ROI localization based finger vein authentication. J Adv Res Dyn Control Syst. 2018

[14] Krizhevsky, Alex., Ilya Sutskever \& Geoffrey E. Hinton. ImageNet Classification with Deep ConvolutionalNeural Networks. ILSVRC. 2012

[15] Maheswari, S. N. Sangeethaa \& P. Uma. An Intelligent Model for Blood Vessel Segmentation in Diagnosing DR. Journal of Medical System, 165175. 2018

[16] Ting, Daniel Shu Wei. M. P., Carol Yim-Lui Cheung, P., Gilbert Lim, P., Gavin SiewWei Tan, F., Nguyen D. Quang, B. Alfred Gan, MSc; Haslina Hamzah, BSc; Renata Garcia-Franco, MD; Ian Yew San Yeo, FRCSEd; Shu Yen Lee, FRCSEd; Edmund Yick Mun Wong, FRCSEd; Charumathi Sabanayagam, MD, PhD; Mani Baskaran, MD, PhD; Farah Ibrahim, MB, BCh, BAO; Ngiap Chuan Tan, MCI, FAMS; Eric A. 
Finkelstein, MHA, PhD; Ecosse L. Lamoureux, PhD; Ian Y. Wong, FRCOph; Neil M. Bressler, MD; Sobha Sivaprasad, FRCOph; Rohit Varma, MD, MPH; Jost B. Jonas, MD, PhD; Ming Guang He, MD, PhD; Ching-Yu Cheng, MD, PhD; Gemmy Chui Ming Cheung, FRCOph; Tin Aung, MD, PhD; Wynne Hsu, PhD; Mong Li Lee, PhD; \& Tien Yin Wong, MD, PhD. Development and Validation of a Deep Learning System. JAMA, 2211-2223. 2017.

[17] Muhammad, Nur Azida., Nasir Amelina Ab., Ibrahim, Zaidah., Sabri, Nurbaity. Evaluation of CNN, Alexnet and GoogleNet for Fruit Recognition. Indonesian Journal of Electrical Engineering and Computer Science. Vol 12. Pp 468-475. November 2018.

[18] Part, Harry., Frans Coenen. Deborah M Broadbent, Simon P Harding \& Yalin Zheng. Convolutional Neural Network for Diabetic Retinopathy. Science Direct, 200-205. 2016.

[19] Salamat, Nadeem., Malik M. \& Saad Missen, Aqsa Rashid. diaetic retinopathy techniques in retinal images: A review. ELSEVIER, 168-188. 2019

[20] Sangeetha S. N. \& P. Uma Maheswari. An Intelligent Model for Blood Vessel Segmentation in Diagnosing DR Using CNN. Journal of Medical Systems, 175-185. 2018.

[21] Stuart J. Russell, Peter Norvig, Artificial Intelligence - A Modern Approach. New Jersey, Simon and Schuster Company. ArticialIntelligence - A Modern Approach. 1995.

[22] Sultana, Farhana., Abu Sufian, Paramartha Dutta. Advandcements in Image Classification using Convolutional Neural Network. International Conference on Research in Computational Intelligence and Communication Networks (ICRCICN), 122-130. 2018.

[23] Swoud L., Hurtut T., Chelbi J., Cheriet F., Pierre Langlois JM (2015) Red lession detection using diabetic retinopathy screening. IEEE transactions on Medical Imaging.

[24] Szegedy, Christian., Wei Liu, Yangqing Jia, Pierre Sermanet, Scott Reed, Dragomir Anguelov, Dumitru Erhan, Vincent Vanhoucke, Andreq Rabinovich. Going Deeper with Convolution. ILSVRC. 2014.

[25] Wan, Azani Mustafa., Aimi Salihah Abdul Nasir, Haniza Yazid. Diabetic Retinopathy (DR) on Retinal Image: A Pilot Study. Journal of Physics Conference Series.2. 20183

[26] Wan, Shaohua., Yan Liang \& Yin Zhang. Deep convolutional neural networks for diabetic retinopathy detection by image classification. ELSEVIER, 274-282. 2018 
[27] Yi-Peng Liua, Z. L. Referable diabetic retinopathy identification from eye fundus images with. ELSEVIER, 1-7. 2019

[28] Shanthi, T., R.S. Sabeenian. Modified Alexnet architecture for classification of diabetic Retinopathy images. ELSEVIER, 56-64. 2019

[29] Xu, Kelvin., Jimmy Lei Ba, Ryan Kiros, Kyunghyun Cho, Aaron Courville, Ruslan Salakhutdino, Richard S. Zemel, Yoshua Bengio. (2015). Show, Attend and Tell: Neural Image Caption Generation with Visual Attention. ICML.

[30] Li, Liu., Mai Xu, Xiaofei Wang, Lai Jiang \& Hanruo Liu. (2019). Attention Based Glaucoma Detection: A Large-scale Database and CNN Model. IEEE Xplore, 10571-10580

\section{Notes on contributors}

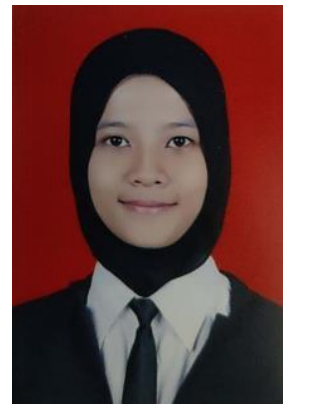

Amnia Salma is student at the Department of Mathematics, Universitas Indonesia, Depok, West Java, Indonesia. Her main research interests include Artifficial Intelligence, image classification, and image preprocessing. She has published several research articles in international journals of mathematics and computer science.

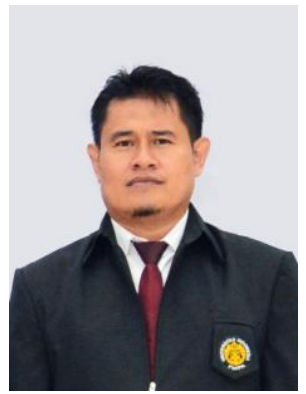

Assoc. Prof. Alhadi Bustamam Ph.D is a lecturer at the Department of Mathematics, Universitas Indonesia, Depok, West Java, Indonesia. He is one of the leading scientist and researcher in the field of data science, bioinformatics, advanced computing, GPU computing and applied computational mathematics. He has been received some highly competitive research grants from Directorate General of Higher Education Indonesian (DIKTI) and Universitas Indonesia including PITTA A, PITTA B, QQ research grants from UI, PUPT, PDUPT, PTUPT, World Class Research, Konsorsium DIKTI, MIT-Indonesian Research Alliance (MIRA) from DIKTI. He has been published more than 100 journals and conference proceedings indexed by Scopus. 


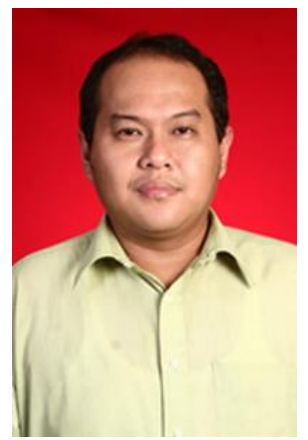

Anggun Rama Yudantha is a vitreoretinal surgeon and medical staff in the Vitreoretinal Department in Universitas Indonesia in Cipto Mangunkusumo Kirana Hospital and Universitas Indonesia Hospital since 2011. $\mathrm{He}$ is a member of Indonesian Doctors Association (IDI), Indonesian Vitreoretinal Society (INAVRS), and he is also currently serving as a member of the Continuing Professionalism Development Commission of Indonesian Ophthalmologist Association (PERDAMI).

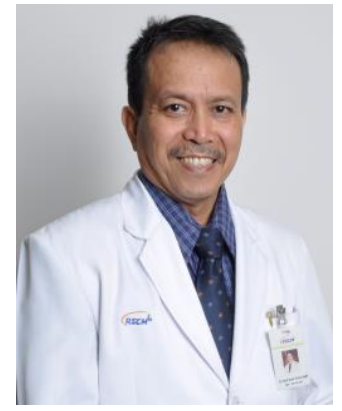

Andi Arus Victor is a vitreoretinal surgeon and head of Vitreoretinal Department in Universitas Indonesia in Cipto Mangunkusumo Kirana Hospital and Universitas Indonesia Hospital. $\mathrm{He}$ is a member of Indonesian Doctors Association (IDI), Indonesian Vitreoretinal Society (INAVRS), and he is also currently serving as a member of the Continuing Professionalism Development Commission of Indonesian Ophthalmologist Association (PERDAMI).

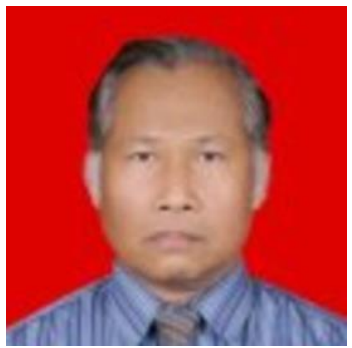

Wibowo Mangunwardoyo is Professor in Biology Department, Universitas Indonesia. His main research interests include Biodiversity, Genetics, and Pathogenesis. He has published several research articles in international journals of biology and biodiversity. 